\title{
Contests with Endogenous Deadlines
}

Citation for published version (APA):

Seel, C. (2018). Contests with Endogenous Deadlines. Journal of Economics \& Management Strategy, 27(1), 119-133. https://doi.org/10.1111/jems.12228

Document status and date:

Published: 01/04/2018

DOI:

10.1111/jems.12228

Document Version:

Publisher's PDF, also known as Version of record

Document license:

Taverne

\section{Please check the document version of this publication:}

- A submitted manuscript is the version of the article upon submission and before peer-review. There can be important differences between the submitted version and the official published version of record.

People interested in the research are advised to contact the author for the final version of the publication, or visit the DOI to the publisher's website.

- The final author version and the galley proof are versions of the publication after peer review.

- The final published version features the final layout of the paper including the volume, issue and page numbers.

Link to publication

\footnotetext{
General rights rights.

- You may freely distribute the URL identifying the publication in the public portal. please follow below link for the End User Agreement:

www.umlib.nl/taverne-license

Take down policy

If you believe that this document breaches copyright please contact us at:

repository@maastrichtuniversity.nl

providing details and we will investigate your claim.
}

Copyright and moral rights for the publications made accessible in the public portal are retained by the authors and/or other copyright owners and it is a condition of accessing publications that users recognise and abide by the legal requirements associated with these

- Users may download and print one copy of any publication from the public portal for the purpose of private study or research.

- You may not further distribute the material or use it for any profit-making activity or commercial gain

If the publication is distributed under the terms of Article $25 \mathrm{fa}$ of the Dutch Copyright Act, indicated by the "Taverne" license above, 


\title{
Contests with endogenous deadlines
}

\author{
Christian Seel (iD
}

Maastricht University,

Department of Economics

(Email: c.seel@maastrichtuniversity.nl)

\begin{abstract}
This paper analyzes the problem of a contest designer who chooses a starting time and a deadline of the contest to maximize discounted total effort by the contestants. Each contestant secretly decides how much effort to exert between the starting time and the deadline. At the deadline, the contestant who exerted most effort wins a prize, which consists of the endowment of the designer and collected interest. The contest has a unique Nash equilibrium. In the main model, the designer should announce the contest immediately with a short deadline to promote intense competition. I analyze how the optimal starting time and deadline change for a variable contest prize, different types of asymmetries, a Tullock lottery contest success function, and different goal functions of the designer.
\end{abstract}

\section{1 | INTRODUCTION}

Contests are frequently used to promote competition, both within firms and between firms or individuals. Many contests share the same temporal structure: the designer chooses when to announce the competition and specifies the assessment rules, the deadline, and the winner prize. All contestants can exert effort between the announcement and the deadline, at which the best performing contestant receives the prize.

Let me provide a few examples, which fit the above description to discuss the scope of this paper. For instance, at schools or universities, the teacher announces the day and the importance of the test and the students decide when and how much they study. The best grade within their peer group is the "prize," which yields prestige and might facilitate applications for the next higher career level or scholarships. The teacher/school faces the following problems: When should the test be written (deadline)? When should the teacher announce the date of the test (starting time)? How does the answer depend on the objective function of the teacher?

In big law firms, promotion decisions are often taken in the form of a contest, that is, several associates compete for the prize of becoming a partner. In this example, the starting time and prize are given by the beginning of the contract and the value of becoming a partner, respectively. Thus, the relevant question is when the firm should take the "making partner" decision.

As a final example, consider an innovation contest, for example, for improving a search algorithm. The designer faces a tradeoff between the expected quality of the algorithm and the deadline of the contest, which is the first date at which she can use it. Thus, she needs to find the starting time/deadline/prize schedule, which maximizes her objective function.

The literature on the optimal duration in contests, however, is very scarce. Contest models such as all-pay auctions or Tullock contests do not model the time dimension at all, whereas other contest models with unobservable actions abstract from discounting and/or assume infinite or exogenous deadlines. ${ }^{1}$

Instead, this paper treats the contest length as a choice variable of the designer. During the contest stage, participants decide when to exert effort, but cannot observe the rival's effort decisions, that is, their interaction remains static. Equilibrium properties

I am indebted to Alex Gershkov and Paul Schweinzer for a myriad of inspiring discussions. Moreover, I would like to thank the reviewers, Philipp Denter, Jörg Franke, Bart Golsteyn, Diego Moreno, Frank Riedel, Nora Szech, Peter Werner, and seminar audiences in Bielefeld, Karlsruhe, Maastricht, and Madrid for valuable remarks and suggestions. 
such as exerted effort and winning probabilities, however, change depending on the contest duration. Thus, unlike the existing static literature, I can study the optimization problem of a contest designer with a focus on the optimal starting time and deadline.

More precisely, I analyze the problem of an impatient contest designer who maximizes discounted expected total effort by two risk-neutral contestants; effort is interpreted as a proxy for output. The designer has a fixed monetary endowment available for the contest. ${ }^{2}$ She chooses when to announce the contest and for how long to run the contest. At the deadline, the contestant who exerted most effort between the announcement and the deadline wins the prize, that is, the endowment and accumulated interest. In case of a tie, the prize is allocated randomly or split equally.

In the first step, I characterize the Nash equilibria of the contest for all starting time/deadline combinations in Propositions $1-3$. For a fixed starting time, a fixed deadline (and a fixed prize), the contest is essentially isomorphic to an all-pay auction with a specific exponential bidding cost and a bid cap.

By changing the starting time and deadline, the designer influences the bid cap and the cost-prize ratio. There are two mitigating effects: a larger deadline increases expected effort, but due to the discounting, it also reduces the valuation of the designer. Propositions 4 and 5 characterize the starting time and deadline which optimally balance the two effects. The main predictions are: (i) it is optimal to announce the contest immediately and (ii) the deadline of the contest should be relatively short. By Proposition 6, these predictions extend if the contest designer also chooses the size of the prize.

The remainder of the paper studies the robustness of the predictions to changes in the main assumptions. By Propositions 7 and 8 , the main predictions extend if contestants differ in their effort cost. In this case, the deadline helps to level the playing field as in Che and Gale (1998), Kirkegaard (2012), and Siegel (2014).

The main predictions also remain valid for a Tullock lottery contest success function. Thus, although the standard static Tullock lottery contest and the static all-pay auction yield qualitatively different equilibria, imposing optimality and taking the time dimension into account establishes a much closer connection between the two contest success functions.

Both main predictions - the optimality of announcing the contest immediately and the relatively short deadline-do not extend if the designer maximizes the highest discounted effort of one participant. This goal function is prevalent in architecture contests, where the contest designer can often only implement one proposal. In this case, an intermediate deadline is sometimes optimal for the designer. Finally, the main results do not extend if the contest designer is more patient than the contestants. In this case, she might still choose a relatively short duration of the contest, but to delay the announcement.

\section{1 | Related literature}

The model can be seen as a modified war of attrition with discounting and a deadline. ${ }^{3}$ As in a standard war of attrition, the player who stays in longer wins the prize and both players incur effort cost independently of the outcome.

Compared to a war of attrition, however, the present paper reverses the informational assumptions, that is, no player can observe his rival. Although a war of attrition captures applications such as animal conflicts (Bishop \& Cannings, 1978; Maynard Smith, 1974) or price wars (Fudenberg \& Tirole, 1986), applications of this model include job promotion contests, competitions for grants, or design competitions, in which research is conducted secretly and the progress of all participants is evaluated at the deadline; see also Taylor (1995) for a more detailed motivation and more applications.

The different informational assumptions lead to a reversed timing structure in equilibrium: players exert effort from a certain time onwards. Hence, the player who starts earliest wins the game, whereas the player who persists longest wins in a war of attrition. The resulting payoffs are pay-your-effort rather than incurring the effort cost of the player who resigns earlier. Finally, differing from a war of attrition which has a myriad of asymmetric equilibria, the present model has a unique equilibrium. Thus, despite a similar game structure, the different informational assumptions reverse many standard results.

Finally, the paper relates to a recent branch of literature on optimal deadlines started by Damiano, Li, and Wing (2012). They analyze a war of attrition with private information and a common interest part and characterize the welfare maximizing deadline. My focus, however, lies on the maximization of expected discounted total effort. Lang, Seel, and Strack (2014) consider a stochastic contest model with discrete jumps and without discounting. They provide a partial ranking of expected total effort.

The rest of the paper is organized as follows. Section 2 introduces the model. In Section 3, I derive the equilibrium of the contest and the optimal starting time/deadline combination for the contest designer. Section 4 is devoted to different extensions of the main model. The results are summarized and discussed in Section 5. Most proofs are relegated to the Appendix.

\section{2 | THE MODEL}

Consider a model with a risk-neutral contest designer and $i=1,2$ risk-neutral contestants. The contest designer decides on the time $\underline{T}$ (starting time) at which she announces the contest and on the time $T$ (deadline) at which the contest ends. The designer has 
an endowment $P$, on which she collects interest at the interest rate $r$ until the deadline $T$. At any point $t \in[\underline{T}, T]$, each contestant decides whether to exert effort $e_{t}^{i}=1$ or not to exert effort $e_{t}^{i}=0$. The effort decisions of each player are unobservable to his rival. Exerting effort induces a flow cost of $c$, whereas no effort induces no flow cost. ${ }^{4}$ The net present value of total cost at time $t=0$ is thus $-\int_{T}^{T} c e_{t}^{i} \exp (-r t) d t^{5}$

At the contest deadline $T$, the designer pays $P \exp (r T)$ to the contestant who exerted most effort. Thus, the net present value at time $t=0$ of winning the prize is $P$. In case of a tie, the prize is randomly allocated or split between the contestants. ${ }^{6}$ The contest designer chooses $\underline{T}$ and $T$ to maximize the expected discounted sum of efforts $\mathbb{E}\left[\exp (-r T) \sum_{i=1}^{2} \int_{\underline{T}}^{T} e_{t}^{i} d t\right]$. I solve the contest using the Nash equilibrium concept, because no new information about the rival's strategy arrives over time.

\section{3 | EQUILIBRIUM ANALYSIS}

In this section, I compute the optimal starting time/deadline combination $(\underline{T}, T)$ for the contest designer. To do so, I first derive different Nash equilibria in the contest depending on the parameters and relate it to all-pay auctions and wars of attrition. Second, I determine the expected discounted total effort for each parameter range in closed form. In the last step, I derive the starting time/deadline combination, which maximizes expected discounted total effort. For notational convenience, I henceforth omit player labels whenever there is no risk of confusion.

\section{1 | Nash equilibria in the contest}

If a contestant exerts effort for a fixed amount of time, due to the discounting, it is cheapest for him to start as late as possible. This directly yields the following lemma which is stated without proof.

Lemma 1 (Delay). If a player exerts effort on some interval ( $s, \tilde{s}]$, then the player also exerts effort for almost all $t \in[\tilde{s}, T]$.

Hence, the decision problem of each player reduces to finding a starting time $s \geq \underline{T}$ such that the player exerts effort at time $t$ if and only if $s \leq t \leq T$.

The next two lemmas are standard results in all-pay models; the proof of Lemma 2 is omitted because it directly follows from the arguments in Baye et al. (1996).

Lemma 2 (No Interior Mass Point). In equilibrium, no player starts with strictly positive probability at a time $\underline{T}<s<T$. At least one player starts before time $T$ with probability 1.

Lemma 3 (Zero Profits). Assume that $\exp (-r \underline{T})-\exp (-r T)>\frac{P r}{2 c}$. In any Nash equilibrium, both players make zero profits.

Roughly speaking, the condition in Lemma 3 ensures the game to be long enough such that always exerting effort might lead to a negative profit. In such cases, no contestant generates any equilibrium profit.

The previous lemmas are helpful in deriving Nash equilibria for three different parameter ranges in the next step. With slight abuse of terminology, I define the contest duration by $\exp (-r \underline{T})-\exp (-r T){ }^{7}$

Proposition 1 (Short Duration). Assume that $\exp (-r \underline{T})-\exp (-r T) \leq \frac{P r}{2 c}$. In the unique Nash equilibrium, both players always exert effort, that is, $s=\underline{T}$.

Proposition 2 (Intermediate Duration). Assume that $\frac{P r}{c}>\exp (-r \underline{T})-\exp (-r T)>\frac{P r}{2 c}$. In the unique Nash equilibrium, each player randomizes his starting time s according to the cumulative distribution function

$$
F(s)= \begin{cases}0 & \text { for all } 0 \leq s<\underline{T} \\ 2\left(1-\frac{c}{P r}(\exp (-r \underline{T})-\exp (-r T))\right) & \text { for all } \underline{T} \leq s<\tilde{s} \\ 1-\frac{c}{P r}(\exp (-r s)-\exp (-r T)) & \text { for all } s \in[\tilde{s}, T] \\ 1 & \text { for all } s>T,\end{cases}
$$

where $\tilde{s}=-\frac{1}{r} \log \left(2 \exp (-r \underline{T})-\exp (-r T)-\frac{P r}{c}\right)$. 
Proposition 3 (Long Duration). Assume that $\frac{P r}{c} \leq \exp (-r \underline{T})-\exp (-r T)$. In the unique Nash equilibrium, each player randomizes the starting time s according to the cumulative distribution function

$$
F(s)= \begin{cases}0 & \text { for all } 0 \leq s \leq \hat{s} \\ 1-\frac{c}{P r}(\exp (-r s)-\exp (-r T)) & \text { for all } s \in(\hat{s}, T] \\ 1 & \text { for all } s>T,\end{cases}
$$

where $\hat{s}=-\frac{1}{r} \log \left(\frac{P r}{c}+\exp (-r T)\right)$.

\section{2 | Equilibria and their relation to the literature}

The model has a close connection to two well-known models from the literature, wars of attrition, and all-pay auctions. Let me first discuss the relation to wars of attrition. For a short duration, both players exert effort during the entire contest, which is also the equilibrium in a war of attrition with a short deadline.

The equilibrium for an intermediate duration is also similar to the symmetric equilibrium of a war of attrition with an intermediate deadline. More precisely, there is a positive probability that players exert effort throughout the entire game; see, for example, Bishop and Cannings (1978) or Hendricks et al. (1988). There is an interior interval in which no player starts with positive probability, which corresponds to the interval in which no player resigns with positive probability in a war of attrition with a deadline.

As for an intermediate duration, players also randomize on an interval to make their rivals indifferent for a long duration. In the latter case, however, no player exerts effort throughout the entire time interval $[\underline{T}, T]$ with positive probability, because this is too costly. This type of equilibrium does not arise in a war of attrition, where previous effort cost become sunk.

The equilibrium construction has an even closer relation to all-pay auctions: suppose players are restricted to choose undominated strategies, that is, they only exert effort between a starting time $s$ and the deadline $T$. The resulting reduced-form game is isomorphic to an all-pay auction with a cost function $c(x)=c \int_{0}^{x} \exp (-r(T-t)) d t$-where a bid $x=T-s$ corresponds to a starting time $s$-and a bid cap at $\bar{x}=T-\underline{T}$.

In their seminal paper, Che and Gale (1998) characterize equilibria for the three corresponding parameter regions for an allpay auction with a bid cap and a linear cost of bidding. The equilibrium construction in this section is a technical extension of Che and Gale (1998) allowing for a specific nonlinear cost of bidding which results from the discounting. ${ }^{8}$

As the interest rate vanishes, the net present value of total cost converges to $\lim _{r \rightarrow 0}-\int_{\underline{T}}^{T} c e_{t}^{i} \exp (-r t) d t=-\int_{\underline{T}}^{T} c e_{t}^{i} d t$ and the total cost of exerting effort for $x$ units of time becomes linear, that is, $\lim _{r \rightarrow 0} c(x)=c \bar{x}$. Thus, as the interest rate goes to zero, the equilibrium effort time distributions converge to the equilibrium bidding distributions in Che and Gale (1998). ${ }^{9}$

If the interest rate equals zero, Lemma 1 is no longer valid and the contestants are indifferent between any distribution with the same total duration of exerting effort. Thus, there are equilibria in which effort is no longer exerted on a connected interval. ${ }^{10}$

The main difference from the standard all-pay auction model is conceptual: the designer's choice of starting time, deadline, and prize affects both the cost structure and the maximal feasible effort level. Thus, despite the static nature of the interaction in the contest, including the time dimension will be important to study the effects of discounting. These effects are absent in the standard static setting and will be illustrated in the next section.

\section{3 | The designer's problem}

We are now ready to tackle the designer's problem: Which starting time/deadline combination maximizes expected discounted equilibrium effort? For a short duration (Proposition 1), discounted total efforts are

$$
\sum_{i=1}^{2} \int_{\underline{T}}^{T} e_{t}^{i} \exp (-r T) d t=2(T-\underline{T}) \exp (-r T) .
$$

A closed-form solution for the other two regions of the parameter space is presented in the appendix. The direct approach would be to maximize $\underline{T}$ and $T$ on all three regions of the parameter space in order to find local maxima and to compare them across regions. This would, however, result in calculations that are difficult to handle analytically for the latter two cases.

Instead, I use a different approach in the next step to show that for any given $T$, the optimal contest involves a $\underline{T}$ such that the resulting duration is short (Proposition 4). In a second step, I derive a closed-form solution for the optimal starting time/deadline combination $(\underline{T}, T)$ (Proposition 5). 
Note that the maximal discounted effort the designer can potentially generate with a deadline $T$ is $2 T \exp (-r T)$. This function attains its maximum at $T^{*}=\frac{1}{r}$. Thus, if both players exert effort throughout the entire contest for $T=\frac{1}{r}$ and $\underline{T}=0$, setting starting time and deadline in this way is the optimal solution. By Proposition 1, such an equilibrium exists and lies in the interior of the range characterized in Proposition 1 if

$$
1-\exp (-r T)<\frac{P r}{2 c}
$$

Plugging $T^{*}=\frac{1}{r}$ into Equation (2), we obtain the following lemma:

Lemma 4. The unique global maximum is attained at an interior solution for a short deadline and $\underline{T}=0$ if $\frac{P r}{2 c}>1-\frac{1}{e}$.

The result is intuitive, because for a high interest rate, the designer wants to get the discovery quickly. Note that there is a qualitative difference in the equilibrium for a short and an intermediate duration: in the latter case, contestants do not spend full effort with probability one. The undiscounted effort for an intermediate duration also increases in $T$, but at a smaller rate than for a short duration. By the next proposition, expected discounted total effort is always maximized for a short duration.

\section{Proposition 4 (Optimal Starting Time).}

(i) If either $\frac{P r}{2 c} \geq 1$ or $\frac{P r}{2 c}<1$ and $T \leq-\frac{1}{r} \log \left(1-\frac{P r}{2 c}\right)$, the starting time $\underline{T}=0$ maximizes total expected discounted effort.

(ii) For $\frac{P r}{2 c}<1$ and $T>-\frac{1}{r} \log \left(1-\frac{P r}{2 c}\right)$, the starting time $\underline{T}=-\frac{1}{r} \log \left(\frac{P r}{2 c}+\exp (-r T)\right)$ maximizes total expected discounted effort.

Proof. Case (i) is trivial: both players exert effort throughout the entire game (see equilibrium for a short duration), that is, effort is maximal.

For Case (ii), note that both players exert effort at any time after $\underline{T}$ in equilibrium. In the following, I show that setting $\underline{T}$ as described in the proposition yields the maximal possible discounted effort of any scheme with maximal transfer $P$ and deadline $T$ which respects the participation constraints. Thereby, it is clearly also the optimal choice for the contest.

Let us maximize total effort subject to the aggregate participation constraint, that is, the sum of the effort cost should not exceed the prize. By Lemma 1, I only need to find the optimal starting times. Thus, I get

$$
\max _{s_{1}, s_{2}}\left(T-s_{1}\right)+\left(T-s_{2}\right)
$$

subject to

$$
\int_{s_{1}}^{T} c \exp (-r t) d t+\int_{s_{2}}^{T} c \exp (-r t) d t \leq P .
$$

The solution to this problem is $s_{1}=s_{2}=-\frac{1}{r} \log \left(\exp (-r T)+\frac{P r}{2 c}\right)$, the starting time $\underline{T}$ in Case (ii).

Thus, to find the optimal $(\underline{T}, T)$-combination for the designer, it remains to find the optimal $T$. Note that if $\operatorname{Pr} \geq 2 c$ or $\operatorname{Pr}<2 c$ and $T \leq-\frac{1}{r} \log \left(1-\frac{\bar{P} r}{2 c}\right)$, the optimization problem is $\max 2 T \exp (-r T)$. On the other hand, for $\operatorname{Pr}\langle 2 c$ and $T>$ $-\frac{1}{r} \log \left(1-\frac{P r}{2 c}\right)$, the optimization problem is $\max 2(T-\underline{T}) \exp (-r T)$. Plugging in the optimal $\underline{T}$ from Proposition 4(ii), I obtain $\max 2\left(T+\frac{1}{r} \log \left(\frac{P r}{2 c}+\exp (-r T)\right)\right) \exp (-r T)$.

Lemma 5. The function $2\left(T+\frac{1}{r} \log \left(\frac{P r}{2 c}+\exp (-r T)\right)\right) \exp (-r T)$ is decreasing in $T$ for all $T \geq-\frac{1}{r} \log \left(1-\frac{P r}{2 c}\right)$.

By Lemma 5, taking the optimal corresponding deadline $T$ as given, expected discounted total effort is decreasing for any duration such that $\underline{T}>0$. This allows us to state the main result about the contest designer:

Proposition 5 (Optimal Starting Time and Deadline). The contest designer chooses the starting time $\underline{T}=0$. If $\frac{P r}{2 c} \geq 1-\frac{1}{e}$, the optimal deadline is $T=\frac{1}{r}$. If $\frac{P r}{2 c}<1-\frac{1}{e}$, the optimal deadline is $T=-\frac{1}{r} \log \left(1-\frac{P r}{2 c}\right)$.

Proof. By Proposition 4, the optimal starting time is $\underline{T}=0$ for Case (i). By Lemma 5, the profit is decreasing in $T$ for the parameters considered in Case (ii) of Proposition 4. Thus, the optimal $T$ in this case is also chosen such that $\underline{T}=0$. Because these cases contain all parameters, I obtain $\underline{T}=0$. 
To prove the second part of the statement, recall that the profit is increasing for a short duration until $T=\frac{1}{r}$. Thus, $T$ is either the interior maximum $T=\frac{1}{r}$ if $\frac{P r}{2 c}>1-\frac{1}{e}$ or the corner solution $T=-\frac{1}{r} \log \left(1-\frac{P r}{2 c}\right)$ otherwise, that is, the maximal $T$ such that $\underline{T}=0$ is optimal.

The optimal deadline in the first case is an interior solution. Intuitively, $T=\frac{1}{r}$ is the point at which the relative increase in total effort is exactly offset by the relative loss due to waiting. The second case is the largest possible deadline that remains within the parameter space of short deadlines (corner solution). Here, increasing the deadline further does not increase expected discounted total effort because the parameter region switches to an intermediate deadline. To sum up, the model yields a clear prediction: it is always optimal to start the contest without any delay and the deadline of the contest should be short enough such that both contestants choose to exert effort throughout the entire contest. This differs from the results in Che and Gale (1998) where a bid cap might increase expenditures only for an asymmetric setting.

\section{4 | Variable prize}

So far, we have assumed that the endowment of the contest designer is fixed. Although this covers applications in which a principal endows the designer with a certain budget, there are other cases in which the designer not only chooses the starting time and deadline, but also has to pay for the prize. Thus, the designer chooses the optimal contest for the goal function

$$
\max _{\underline{T}, T, P} \exp (-r T) \mathbb{E}\left(\sum_{i=1}^{2} \int_{\underline{T}}^{T} e_{i}^{t} d t\right)-P .
$$

For this goal function, we obtain the following characterization.

Proposition 6. Suppose the contest designer maximizes Equation (3).

(i) If $c \geq 1$, any contest with a prize $P>0$ yields a negative value of the designer's goal function. Thus, the designer should not hold a contest at all.

(ii) If $c<1$, the optimal solution is given by $P=(1-\exp (c-1)) \frac{2 c}{r}, \underline{T}=0$ and $T=\frac{1-c}{r}$. The resulting profit of the designer is $\Pi=\frac{2}{r}(\exp (c-1)-c)>0$.

Proof. Proposition 5 contains the optimal starting time and deadline for every given prize. Thus, taking the resulting values as given, it remains to find the optimal prize. Note that we only need to consider values of $P$ such that $\frac{P r}{2 c} \leq 1-\frac{1}{e}$, because higher prizes would not increase effort by Proposition 5. Thus, plugging $T=-\frac{1}{r} \log \left(1-\frac{P r}{2 c}\right)$ and $\underline{T}=0$ into Equation (3) yields

$$
\Pi(P)=-\frac{2}{r} \log \left(1-\frac{P r}{2 c}\right)\left(1-\frac{P r}{2 c}\right)-P
$$

The first-order condition is

$$
\frac{d \Pi(P)}{d P}=\frac{1}{c}\left(1+\log \left(1-\frac{P r}{2 c}\right)\right)-1=0,
$$

which reduces to $P=(1-\exp (c-1)) \frac{2 c}{r}$.

Thus, $P$ is positive if and only if $c<1$ (the fact that $P$ is the maximum follows from the second-order condition or a sign test). This establishes Part ( $i)$ of the proposition. For Part (ii), plug the value for $P$ back into the expression for $T$ to obtain $T=\frac{1-c}{r}$. Plugging $P$ and $T$ into the goal function of the designer, I get $\frac{2}{r}(\exp (c-1)-c)>0$.

The contest designer does not treat the prize as given as in the previous section, but bears the cost of a prize increase himself. Thus, she has to balance the higher induced effort against the cost of providing a higher prize. If effort costs are too high as in (i), any contest with a positive prize results in a loss, that is, $P=0$ (no contest) is optimal. If the optimal contest yields a positive profit, the resulting deadline is short and reduces even further compared to Proposition 5 , because $T=\frac{1-c}{r}<\frac{1}{r}$. 


\section{4 | EXTENSIONS}

In this section, I consider how robust the predictions about the optimal contest starting time and deadline are to different changes in the model.

\section{1 | Asymmetric cost functions}

As in the baseline model, two players compete for the prize as described in Section 2. Differing from that model, however, players now have different flow costs, without loss of generality $c_{1}<c_{2}$.

The reasoning in the proof of Proposition 1 implies that there is a (unique) Nash equilibrium in which both players exert effort throughout the entire contest if this leads to a (strictly) positive profit for both players. If this condition is satisfied for player 2 , then it is also satisfied for player 1 who has lower flow cost. ${ }^{11}$ Thus, we obtain:

Proposition 7. Assume that $\exp (-r \underline{T})-\exp (-r T) \leq \frac{P r}{2 c_{2}}$. In the Nash equilibrium, both players always exert effort, that is, $s=\underline{T}$.

The following proposition extends the main result to asymmetric costs.

Proposition 8. The contest designer chooses the starting time $\underline{T}=0$. If $\frac{P r}{2 c_{2}} \geq 1-e$, the optimal deadline is $T=\frac{1}{r}$. If $\frac{P r}{2 c_{2}}<$ $1-e$, the optimal deadline is $T=-\frac{1}{r} \log \left(1-\frac{P r}{2 c_{2}}\right)$.

Establishing optimality of a short duration is more complicated than in the symmetric setting, because the optimal contest is no longer a first-best scheme as in the proof of Proposition 4. Instead, the proof of Proposition 8 derives the equilibrium distributions for all possible parameters and bounds the corresponding expected efforts using results from the symmetric setting. Intuitively, in the asymmetric case, a short duration has the additional benefit of leveling the playing field between unequal contestants as in Che and Gale (1998) and thus remains optimal.

\section{2 | Tullock lottery contest success function}

So far, I have assumed that the player who exerts most effort wins the contest with probability 1 . I now extend the analysis to a Tullock lottery contest success function, that is, each player's probability of winning the contest is proportional to his share in the total effort (with the usual convention that the winning probability is $\frac{1}{2}$ if total effort is 0 ).

As before, due to the discounting, the optimal decision of a player reduces to finding a starting time $s_{i} \geq \underline{T}$ such that $e_{i}^{t}=1$ if and only if $t \geq s_{i}$. The optimization problem of player $i$ thus simplifies to

$$
\max _{s_{i}} P \frac{T-s_{i}}{2 T-s_{i}-s_{j}}-\int_{s_{i}}^{T} c \exp (-r t) d t
$$

The first derivative of this function is

$$
\frac{d \pi_{i}}{d s_{i}}=P \frac{s_{j}-T}{\left(2 T-s_{i}-s_{j}\right)^{2}}+c \exp \left(-r s_{i}\right)
$$

If $\underline{T}=0$, always effort by both players is the Nash equilibrium if nobody benefits from increasing his starting time: ${ }^{12}$

$$
\frac{d \pi_{i}}{d s_{i}}\left(s_{i}, s_{j}=0\right) \leq \frac{d \pi_{i}}{d s_{i}}\left(s_{i}=0, s_{j}=0\right)=P \frac{-T}{(2 T)^{2}}+c \leq 0, \text { that is, if } \frac{P}{c T} \geq 4 .
$$

If always effort by both players is not a Nash equilibrium, I obtain the symmetric Nash equilibrium by setting

$$
\frac{d \pi_{i}}{d s_{i}}\left(s_{1}=s_{2}=s\right)=P \frac{s-T}{(2 T-2 s)^{2}}+c \exp (-r s)=0
$$

which yields

$$
\exp (-r s)(T-s)=\frac{P}{4 c} .
$$


Thus,

$$
s=\frac{r T-W\left(\frac{P \exp (r T) r}{4 c}\right)}{r},
$$

where $W$ denotes the Lambert $W$ Function.

Unlike the all-pay contest success function, a Tullock lottery contest success function always admits a (unique) symmetric pure-strategy equilibrium in which both contestants exert effort after a certain starting time. Thus, a restriction on $\underline{T}$ (weakly) reduces effort. Hence, without loss of optimality, I henceforth set $\underline{T}=0$. To find the global maximum, I only need to compare the effort on two intervals: for $T \leq \frac{P}{4 c}$, the effort of each contestant is $\bar{T}$, whereas for $T>\frac{P}{4 c}$, the effort of each contestant is $T-s=$ $\frac{W\left(\frac{P \exp (r T) r}{4 c}\right)}{r}$. For the first interval, I obtain $T^{*}=\frac{1}{r}$ as before. For the second interval, one has to maximize $\frac{W\left(\frac{P \exp (r T) r}{4 c}\right)}{r} \exp (-r T)$. The first derivative of this function with respect to $T$ is

$$
-\frac{r \exp (-r T) W\left(\frac{P \exp (r T) r}{4 c}\right)^{2}}{W\left(\frac{P \exp (r T) r}{4 c}+1\right)}<0,
$$

because $W(x)>0$ for all $x>0$. Thus, it is never optimal to choose $T>\frac{P}{4 c}$. Summing up, I obtain the following proposition:

Proposition 9. The optimal starting time is $\underline{T}=0$. The optimal deadline is $T=\frac{1}{r}$ if $\frac{1}{r}<\frac{P}{4 c}$ and $T=\frac{P}{4 c}$ otherwise.

The optimal equilibrium for a Tullock contest success function is qualitatively similar to the one for the all-pay contest success function, with small differences in the parameter ranges.

\section{3 | Different goal functions of the contest designer}

The related literature mainly focuses on two different goal functions of the contest designer: maximizing expected total effort as analyzed in the main part and maximizing the expected maximum effort; see, for example, Taylor (1995), Moldovanu and Sela (2001), or Seel and Wasser (2014). The latter goal function describes applications such as architecture contests, where the contest designer only implements one of the submitted ideas. The next proposition provides a characterization, which shows that short deadlines need not be optimal in this case.

Proposition 10. Suppose the contest designer maximizes the discounted expected maximum effort $\exp (-r T)$ $\left\{\mathbb{E}\left(\max \left\{\int_{T}^{T} e_{1}^{t} d t, \int_{T}^{T} e_{2}^{t} d t\right\}\right)\right\}$. The maximal discounted effort is obtained for $\underline{T}=0$ and a short deadline if and only if $\frac{P r}{2 c} \geq 1-\frac{1}{e}$.

For $\frac{P r}{2 c} \geq 1-\frac{1}{e}$, the global maximum is still attained for a short deadline with $\underline{T}=0$. On the other hand, for $\frac{P r}{2 c}<1-\frac{1}{e}$, discounted total effort continues to increase as the parameters switch from a short to an intermediate deadline. Intuitively, at an intermediate deadline, there is still a high likelihood that at least one player exerts effort throughout the entire contest.

As another application, consider arms races during wars. Here, (prize) money might only play a secondary role. As such, consider a contest designer who lexicographically cares first about maximizing total effort and second about her costs. To generate the maximal effort for a given time frame $[0, T]$, the designer needs to choose $\underline{T}=0$ and $P \geq \frac{2 c}{r}(1-\exp (-r T))$. Taking the lexicographic preference for a lower prize into account, we obtain $P=\frac{2 c}{r}(1-\exp (-r T))$. Intuitively, because agents already exert maximal effort at that value, increasing the prize further does not increase effort.

\section{4 | Different discount factors}

Up to now, I have implicitly assumed that the discount rate of the contest designer satisfies $\delta=\frac{1}{1+r}$. If she is less patient, that is, $\delta<\frac{1}{1+r}$, the qualitative results of the paper continue to hold. Intuitively, a short deadline becomes even more attractive, because the contest designer urgently needs the output. On the other hand, if the contest designer is more patient, that is, $\delta>\frac{1}{1+r}$, the results might break down: keeping the duration of the contest constant, a later starting time means lower costs for the same prize (both discounted back to $T=0$ ). In turn, competition ensures that contestants exert effort for a longer time period in equilibrium. In the main model, this effect is more than offset by the increased waiting time for the designer. But if her patience is sufficiently 
high, the effect from reducing cost for the contestants becomes dominant. In this case, a contest with a positive starting time and a short duration is optimal.

For the contestants, I have also assumed that their effort costs shrink at the discount rate. There are several reasons why (perceived) effort costs are decreasing over time. For instance, for innovation contests, think about technological progress or a lower opportunity cost in the future due to urgent upcoming deadlines for other projects. For competitions in schools, students might perceive future effort cost lower and procrastinate. The main results continue to hold as long as effort costs shrink, but at most at the speed of the discount rate. If effort costs shrink faster than the discount rate, for example, due to rapid technological progress, the designer might prefer to delay announcing the contest.

\section{5 | DISCUSSION}

The main conceptual contribution of the paper consists of setting up the model and recognizing that (i) the contest is a war of attrition in which participants cannot observe each other and (ii) the strategic incentives are isomorphic to those in an all-pay auction with a bid cap and a specific nonlinear cost of bidding. The equilibrium derivation for the contest stage provides a small technical extension of Che and Gale (1998). More important, in contrast to the bid cap in Che and Gale (1998), the contest starting time and deadline influence the cost structure due to discounting.

In Che and Gale (1998), a bid cap can only lead to an increase in lobbying expenditures-their interpretation for the bidfor asymmetric valuations. Here, the discounting makes a short duration optimal for the contest designer for symmetric and asymmetric marginal cost.

From an applied point of view, Kaplan and Wettstein (2006) criticize that constraints on lobbying expenditures as modeled in Che and Gale (1998) are difficult to monitor and their results do not extend for a nonbinding cap. For the applications in this paper such as examinations in schools and tenure decisions in law firms, a similar critique seems less appealing, because a binding deadline for tests in schools or tenure decisions can be easily enforced.

Finally, let me mention two testable predictions of the model. For examinations in schools, empirical results show the females perform better on average than males. Data-driven explanations include different valuations for winning (Chen, Ong, and Sheremeta, 2015) or a difference in self-discipline Duckworth and Seligman (2006), that is, the perceived time-discount rate. Thus, if skills are homogeneous, this gender gap should disappear for a sufficiently short exam-relevant period.

In architecture contests, the designer often only implements the best proposal. Such contests are predicted to have longer deadlines compared to contests in which total effort is important for the designer.

\section{ENDNOTES}

${ }^{1}$ For the former category, see, for example, Tullock (1980), Hillman and Samet (1987), Hillman and Riley (1989), Baye, Kovenock, and de Vries (1996), Konrad (2002), Siegel (2009a,b), and Alcalde and Dahm (2010); for the latter category, see, for example, Taylor (1995) and Seel and Strack (2013, 2016).

${ }^{2}$ This assumption is relaxed in Section 3.4.

${ }^{3}$ The war of attrition was introduced by Maynard Smith (1974) and has been extensively studied since then; seminal papers include Hendricks, Weiss, and Wilson (1988), Krishna and Morgan (1997), and Bulow and Klemperer (1999).

${ }^{4}$ The main results extend if-instead of the binary effort choice-each player picks an effort intensity $\lambda$ at a linear $\operatorname{cost} c(\lambda)=c \lambda$ with $\lambda \in[0, \bar{\lambda}]$ at any point in time.

${ }^{5}$ Section 4.4 contains a detailed discussion about the implied assumptions on discounting.

${ }^{6}$ If the prize is split, the main results extend for risk-averse contestants.

${ }^{7} \mathrm{I}$ use the term duration, because the term increases in $T$ and decreases in $T$, but contrary to the standard use, the duration is not equal to $T-\underline{T}$.

${ }^{8}$ To the best of my knowledge, there is no direct result in the literature, which covers a general class of cost functions in the complete information case. A treatment for nonlinear cost functions in the incomplete information case is presented in Gavious, Moldovanu, and Sela (2002). The recent literature on all-pay auctions with bid caps considers other extensions of Che and Gale (1998): Szech (2015) considers different tie-breaking rules and Hart (2016) introduces caps of unequal size to the general class of Lotto games.

${ }^{9}$ Che and Gale (1998) allow for heterogeneous valuations. This case is covered by Section 4.1, where the valuations $v_{i}$ in Che and Gale (1998) correspond to $v_{i}=\frac{P}{c_{i}}$ here.

${ }^{10}$ For a negative interest rate $r$, that is, if effort today is less costly than effort tomorrow, Lemma 1 reverses and contestants start to exert effort as early as possible and instead choose a time at which they stop. The rest of the derivation proceeds along the same lines as the paper. 
${ }^{11}$ The set of equilibria for the boundary case $\exp (-r \underline{T})-\exp (-r T)=\frac{P r}{2 c_{2}}$ differs from the symmetric setting: there is continuum of Nash equilibria in which player 1 exerts full effort with probability one and player 2 mixes between no effort and full effort. In line with the standard convention from the industrial organization literature, I select the full effort equilibrium for this case.

${ }^{12}$ Existence and uniqueness of all equilibria in this section follows from Theorem 1 in Szidarovszky and Okuguchi (1997).

\section{ORCID}

Christian Seel (D) http://orcid.org/0000-0001-8450-2048

\section{REFERENCES}

Alcalde, J., \& Dahm, M. (2010). Rent seeking and rent dissipation: A neutrality result. Journal of Public Economics, 94, 1-7.

Baye, M., Kovenock, D., \& de Vries, C. (1996). The all-pay auction with complete information. Economic Theory, 8, 291-305.

Bishop, T., \& Cannings, C. (1978). A generalized war of attrition. Journal of Theoretical Biology, 70, 85-124.

Bulow, J., \& Klemperer, P. (1999). The generalized war of attrition. American Economic Review, 89, 175-189.

Che, Y.-K., \& Gale, I. (1998). Caps on political lobbying. American Economic Review, 88, 643-651.

Chen, Z., Ong, D., \& Sheremeta, R. M. (2015). The gender difference in the value of winning. Economics Letters, 137, $226-229$.

Damiano, E., Li, H., \& Wing, S. (2012). Optimal deadlines for agreements. Theoretical Economics, 7, 357-393.

Duckworth, A. L., \& Seligman, M. E. P. (2006). Self-discipline gives girls the edge: Gender in self-discipline, grades and achievement test scores. Journal of Educational Psychology, 98, 198-208.

Fudenberg, D., \& Tirole, J. (1986). A theory of exit in duopoly. Econometrica, 54, 943-960.

Gavious, A., Moldovanu, B., \& Sela, A. (2002). Bid costs and endogeneous bid caps. RAND Journal of Economics, 33, 709-722.

Hart, S. (2016). Allocation games with caps: from captain lotto to all-pay auctions. International Journal of Game Theory, 45, 37-61.

Hendricks, K., Weiss, A., \& Wilson, C. (1988). The war of attrition in continuous time with complete information. International Economic Review, 29, 663-680.

Hillman, A., \& Riley, J. (1989). Politically contestable rents and transfers. Economics and Politics, 1, 17-39.

Hillman, A., \& Samet, D. (1987). Dissipation of contestable rents by small numbers of contenders. Public Choice, 54, 63-82.

Kaplan, T. R., \& Wettstein, D. (2006). Caps on political lobbying: Comment. American Economic Review, 96, 1351-1354.

Kirkegaard, R. (2012). Favoritism in asymmetric contests: Head starts and handicaps. Games and Economic Behavior, 76, 226-248.

Konrad, K. (2002). Investment in the absence of property rights;: The role of incumbency advantages. European Economic Review, 46, $1521-1537$.

Krishna, V., \& Morgan, J. (1997). An analysis of the war of attrition and the all-pay auction. Journal of Economic Theory, 72, $343-362$.

Lang, M., Seel, C., \& Strack, P. (2014). Deadlines in stochastic contests. Journal of Mathematical Economics, 52, 134-142.

Maynard Smith, J. (1974). The theory of games and evolution of animal conflicts. Journal of Theoretical Biology, 47, $209-221$.

Moldovanu, B., \& Sela, A. (2001). The optimal allocation of prizes in contests. American Economic Review, 91, 542-558.

Seel, C., \& Strack, P. (2013). Gambling in contests. Journal of Economic Theory, 148, 2033-2048.

Seel, C., \& Strack, P. (2016). Continuous time contests with private information. Mathematics of Operations Research, 41, $1093-1107$.

Seel, C., \& Wasser, C. (2014). On optimal head starts in all-pay auctions. Economics Letters, 124, 211-214.

Siegel, R. (2009a). All-pay contests. Econometrica, 77, 71-92.

Siegel, R. (2009b). Asymmetric contests with conditional investments. American Economic Review, 100, 2230-2260.

Siegel, R. (2014). Asymmetric contests with head starts and non-monotonic costs. American Economic Journal: Microeconomics, 6, 59-105.

Szech, N. (2015). Tie-breaks and bid-caps in all-pay auctions. Games and Economic Behavior, 92, 138-149.

Szidarovszky, F., \& Okuguchi, K. (1997). On the existence and uniqueness of pure Nash equilibrium in rent-seeking games. Games and Economic Behavior, 18, 135-140.

Taylor, C. (1995). Digging for golden carrots: an analysis of research tournaments. American Economic Review, 85, 872-890.

Tullock, G. (1980). Efficient rent seeking. In J. Buchanan, R. Tollison, \& G. Tullock (Eds.), Toward a theory of the rent-seeking society, pp. 97-112. College Station, TX: Texas A\&M University Press.

How to cite this article: Seel C. Contests with endogenous deadlines. J Econ Manage Strat. 2018;27:119-133. https://doi.org/10.1111/jems.12228 


\section{APPENDIX}

Proof of Lemma 3. There exists no equilibrium in which both players start at $s=\underline{T}$ with positive probability and at least one player starts at $s=\underline{T}$ with probability 1 , because this would entail a negative profit for the other player. By Lemma 2, at least one player places no mass point/atom at $s=T$ and no player places a mass point in $(\underline{T}, T)$. Combining the two previous statements, we see that starting at the supremum of the starting times $\bar{s}=\sup \left\{s: \exists i\right.$ s.t. $\left.\overline{F_{i}}(s)<1\right\}$ loses with probability 1 . Thus, by continuity, player $i$, without loss of generality player 2 , gets a profit of (at most) zero.

By standard arguments, both players randomize their starting times with a positive density on the same intervals (otherwise, one player could increase his starting time on that interval and obtain the same winning probability at a lower cost).

Case 1: Exactly one player starts with positive probability at $s=\underline{T}$.

In this case, there exists an $\epsilon>0$ such that the rival does not start in an interval $(\underline{T}, \underline{T}+\epsilon)$ with positive probability, because starting at $T$ instead would increase the expected profit. Hence, the first player has an incentive to start at $s=\underline{T}+\frac{\epsilon}{2}$ instead of $s=\underline{T}$, because both starting times guarantee him to win with probability 1 , but the latter one induces a higher cost. This contradicts the equilibrium assumption.

Case 2: Both players start with positive probability $m \in(0,1)$ at $s=\underline{T}$.

Towards a contradiction assume that player 1 makes a positive profit. This entails $F_{1}(T)>F_{2}(\underline{T})$, because player 1 has a higher equilibrium profit. By the argument in Case 1, there exists an $\epsilon>0$ such that no player starts with positive probability in $(\underline{T}, \underline{T}+\epsilon)$. Because both players randomize with positive density on the same intervals, I can define $\tilde{s}=\inf \left\{s: F_{i}(s)>F_{i}(\underline{T})\right\}$. By optimality and continuity, both players are indifferent between starting at $\underline{T}$ and at $\tilde{s}$, which leads to a contradiction, because the gain of player 2 from starting at $\underline{T}$ instead of $\tilde{s}$ is higher, because $F_{1}(\underline{T})>F_{2}(\underline{T})$.

Case 3: No player starts with positive probability at $s=\underline{T}$.

Toward a contradiction assume that player 1 makes a positive profit, in particular by choosing the lowest starting time contained in the support of his randomization. Thus, player 2 also makes a positive profit by starting at the lowest starting time in the randomization of player 1, because it guarantees him to win with probability 1 incurring the same cost as player 1. This contradicts the equilibrium assumption.

Proof of Proposition 1. Existence: If both players exert effort during the entire game, both win the prize with probability $\frac{1}{2}$. For any (pure strategy) deviation, a player wins the prize with probability 0 , because the rival exerts more effort in this case. Thus, the best possible deviation is to exert no effort at all, which leads to a profit of 0 . The equilibrium profit is greater or equal to the profit from the best deviation if

$$
\frac{P}{2}-\int_{\underline{T}}^{T} c \exp (-r t) d t \geq 0 .
$$

This equation simplifies to $\exp (-r \underline{T})-\exp (-r T) \leq \frac{P r}{2 c}$.

Uniqueness: There exists no equilibrium in which a player starts at a time $\underline{T}<s<T$ with positive probability by Lemma 2 . Toward a contradiction, consider an equilibrium in which at least one player does not start at $s=\underline{T}$ with probability 1 . Then, by Lemma 2, the supremum of the starting times of one player loses with probability 1 , that is, by continuity, the profit at least one player, say player 2 , is zero. I distinguish two cases:

Case 1: Player 1 starts at time $t=\underline{T}$ with probability 1.

As I have argued in the existence part, it is not optimal for player 2 to start with positive probability in $(\underline{T}, T)$ against the strategy of player 1 . Thus, the remaining candidates for an equilibrium strategy of player 2 place mass $m \in[0,1)$ at $s=\underline{T}$ (full effort) and $1-m$ at $s=T$ (no effort). Note that no effort leads to strictly lower profit for player 2 if $\exp (-r \underline{T})-\exp (-r T)<\frac{P r}{2 c}$. Thus, in this case, $m=1$ is a profitable deviation.

To complete the proof of Case 1, we have to rule out a different equilibrium for the boundary case $\exp (-r \underline{T})-\exp (-r T)=\frac{P r}{2 c}$ and $m<1$. Compare the profit of player 1 from $s=\underline{T}$ to $s=T-\epsilon$ to get

$$
\pi^{1}(T-\epsilon)-\pi^{1}(\underline{T})=(1-m) P-\int_{T-\epsilon}^{T} c \exp (-r t) d t-\left(1-\frac{m}{2}\right) P+\int_{\underline{T}}^{T} c \exp (-r t) d t .
$$


We can simplify this equation and then plug in $c=\frac{P r}{2(\exp (-r \underline{T})-\exp (-r T))}$ (rewritten expression for the boundary case) to obtain

$$
\pi^{1}(T-\epsilon)-\pi^{1}(\underline{T})=\frac{P}{2}\left(\frac{\exp (-r \underline{T})-\exp (-r(T-\epsilon))}{\exp (-r \underline{T})-\exp (-r T)}-m\right) .
$$

As $\epsilon \rightarrow 0$, the fraction in the brackets of Equation (A1) converges to 1. Thus, by continuity, for any $m \in[0,1)$, there exists an $\epsilon>0$, such that starting at $s=T-\epsilon$ is strictly better for player 1 than starting at $s=\underline{T}$. This contradicts the initial assumption that player 1 starts at $\underline{T}$ with probability 1.

Case 2: Player 1 does not start at $t=\underline{T}$ with probability 1.

Recall that player 2 makes zero profit for his supposed equilibrium strategy. Consider the deviation which starts at time $t=\underline{T}$ for player 2 . This strategy guarantees a winning probability above $\frac{1}{2}$, because player 1 does not start at time $t=\underline{T}$ with probability 1 . Hence,

$$
\pi^{2}(s=\underline{T})>\frac{P}{2}-\int_{\underline{T}}^{T} c \exp (-r t) d t \geq 0
$$

that is, player 2 has a profitable deviation.

Proof of Proposition 2. If a player does not start at $s=\underline{T}$ with positive probability, the other player can start at $s=\underline{T}$ and make a positive profit, which violates Lemma 3. Hence, both players have to start at $s=\underline{T}$ with positive probability. This entails zero profits if

$$
\int_{\underline{T}}^{T} c \exp (-r t) d t=P\left(\frac{F(\underline{T})}{2}+1-F(\underline{T})\right)=P\left(1-\frac{F(\underline{T})}{2}\right) .
$$

Thus,

$$
F(\underline{T})=2\left(1-\frac{c}{r P}(\exp (-r \underline{T})-\exp (-r T))\right)
$$

Because a player who starts at $s>\underline{T}$ can only win against a player who starts after $\underline{T}$, no player starts for $s \in(\underline{T}, \tilde{s})$, because

$$
P(1-F(s))-\int_{S}^{T} c \exp (-r t) d t \leq P(1-F(\underline{T}))-\int_{S}^{T} c \exp (-r t) d t<0 .
$$

For $s \in[\tilde{s}, T]$, the zero profit condition implies

$$
P(1-F(s))-\int_{s}^{T} c \exp (-r t) d t=0
$$

Rearranging, I obtain

$$
F(s)=1-\frac{c}{r P}(\exp (-r s)-\exp (-r T))
$$

Proof of Proposition 3. By Lemma 3, both players make zero profits. Moreover, they randomize with positive density on the same intervals. This uniquely determines the equilibrium distributions for $s \geq \hat{s}$ by Equation (A2). No player has an incentive to start at a time $s<\hat{s}$, because the costs exceed the prize in this case.

\section{Equilibrium efforts for an intermediate or long duration}

To obtain the values for an intermediate duration, I derive expected efforts for the case considered in Proposition 2:

$$
\mathbb{E}\left(\sum_{i=1}^{2} \int_{\underline{T}}^{T} e_{t}^{i} \exp (-r T) d t\right)=2 \exp (-r T)\left(2\left(1-\frac{c}{r P}(\exp (-r \underline{T})-\exp (-r T))\right)(T-\underline{T})+\int_{\tilde{s}}^{T} f(t)(T-t) d t\right)
$$


Note that

$$
\int_{\tilde{s}}^{T} f(t)(T-t) d t=\frac{c}{p} \int_{\tilde{s}}^{T} \exp (-r t)(T-t) d t
$$

Integration by parts yields

$$
\frac{c}{P r}\left[\left(2-\exp (-r T)-\frac{P r}{c}\right)\left(T+\frac{1}{r} \log \left(2-\exp (-r T)-\frac{P r}{c}\right)-\frac{1}{r}\right)+\frac{1}{r} \exp (-r T)\right] .
$$

Thus, the expected discounted sum of efforts which the designer collects for an intermediate duration is given by

$$
\begin{aligned}
\Pi(T)= & \sum_{i=1}^{2} \int_{0}^{T} e_{t}^{i} \exp (-r T) d t=2 \exp (-r T)\left(2\left(1-\frac{c}{r P}(\exp (-r \underline{T})-\exp (-r T))\right)(T-\underline{T})\right. \\
& \left.+\frac{c}{P r}\left[\left(2-\exp (-r T)-\frac{P r}{c}\right)\left(T+\frac{1}{r} \log \left(2-\exp (-r T)-\frac{P r}{c}\right)-\frac{1}{r}\right)+\frac{1}{r} \exp (-r T)\right]\right) .
\end{aligned}
$$

It remains to consider the discounted sum of efforts for the equilibrium for a long duration, which I derived in Proposition 3:

$$
\Pi(T)=\sum_{i=1}^{2} \int_{\hat{s}}^{T} e_{t}^{i} \exp (-r T) d t=2 \exp (-r T)\left(T-\frac{1}{r}+\frac{c}{P r}\left(T \exp (-r T)+\frac{1}{r} \log \left(\frac{P r}{c}+\exp (-r T)\right)\left(\frac{P r}{c}+\exp (-r T)\right)\right)\right) .
$$

Proof of Lemma 5. We have to show that the function

$$
\Pi(T)=2\left(T+\frac{1}{r} \log \left(\frac{P r}{2 c}+\exp (-r T)\right)\right) \exp (-r T)
$$

is decreasing in $T$ for all $T \geq-\frac{1}{r} \log \left(1-\frac{P r}{2 c}\right)$.

The first derivative of this function (dropping the 2 which is irrelevant for the sign of the derivative) is given by

$$
\frac{d \Pi(T)}{d T}=\exp (-r T)\left(1-\frac{\exp (-r T)}{\frac{P r}{2 c}+\exp (-r T)}-r T-\log \left(\frac{\operatorname{Pr}}{2 c}+\exp (-r T)\right)\right)
$$

This derivative is negative if and only if the second term $g(T)=1-\frac{\exp (-r T)}{\frac{P r}{2 c}+\exp (-r T)}-r T-\log \left(\frac{P r}{2 c}+\exp (-r T)\right)$ is negative. Because it is not straightforward to see this from the equation, I proceed in two steps: First, I show that $g(T)$ is negative at the minimal admissible value of $T$. In the second step, $g(T)$ is shown to be negative for all $T$ above the minimal value.

Step 1: At $T=-\frac{1}{r} \log \left(1-\frac{P r}{2 c}\right)$, the equation reduces to

$$
g(T)=\left(\frac{P r}{2 c}+\log \left(1-\frac{P r}{2 c}\right)\right)<0 .
$$

Step 2: The derivative of $g(T)$ is given by

$$
\frac{d g(T)}{d T}=r\left[-1+\exp (-r T)\left(\frac{\frac{P r}{2 c}}{\left(\frac{P r}{2 c}+\exp (-r T)\right)^{2}}+\frac{1}{\frac{P r}{2 c}+\exp (-r T)}\right)\right] .
$$

Rearranging, we obtain

$$
\frac{d g(T)}{d T}=r\left[-1+\frac{\exp (-r T) \frac{P r}{c}+\exp (-2 r T)}{\exp (-r T) \frac{P r}{c}+\exp (-2 r T)+\left(\frac{P r}{2 c}\right)^{2}}\right]<0,
$$

because the fraction is smaller than 1.

Thus, $g(T)$ is negative at $T=-\frac{1}{r} \log \left(1-\frac{P r}{2 c}\right)$ and decreasing afterward, that is, $g(T)$ is negative for all $T \geq-\frac{1}{r} \log \left(1-\frac{P r}{2 c}\right)$. Hence, $\frac{d \Pi(T)}{d T}$ is negative, which means that $\Pi(T)$ is decreasing in $T$ for all $T \geq-\frac{1}{r} \log \left(1-\frac{P r}{2 c}\right)$. 
Proof of Proposition 8. The proof is split up into two steps.

Step 1: I first derive the equilibrium distributions for an intermediate and long duration in Lemmas 6 and 7. In both cases, I omit the uniqueness part in the proof, because it proceeds along the lines of the proof for symmetric cost functions: first, establish that the profits of both players are fixed in equilibrium. Then find the unique distributions which yield these profits for any starting time in the support and lower profits for any other starting time.

Lemma 6. Assume that $\frac{P r}{c_{2}}>\exp (-r \underline{T})-\exp (-r T)>\frac{P r}{2 c_{2}}$. In the unique Nash equilibrium, players randomize their starting times according to the cumulative distribution functions

$$
F_{1}(s)= \begin{cases}0 & \text { for all } 0 \leq s<\underline{T} \\ 2\left(1-\frac{c_{2}}{P r}(\exp (-r \underline{T})-\exp (-r T))\right) & \text { for all } \underline{T} \leq s<\tilde{s} \\ 1-\frac{c_{2}}{P r}(\exp (-r s)-\exp (-r T)) & \text { for all } s \in[\tilde{s}, T] \\ 1 & \text { for all } s>T\end{cases}
$$

and

$$
F_{2}(s)= \begin{cases}0 & \text { for all } 0 \leq s<\underline{T} \\ 2\left(\frac{c_{1}}{c_{2}}-\frac{c_{1}}{P r}(\exp (-r \underline{T})-\exp (-r T))\right) & \text { for all } \underline{T} \leq s<\tilde{s} \\ \frac{c_{1}}{c_{2}}-\frac{c_{1}}{P r}(\exp (-r s)-\exp (-r T)) & \text { for all } s \in(\tilde{s}, T) \\ 1 & \text { for all } s \geq T,\end{cases}
$$

where $\tilde{s}=-\frac{1}{r} \log \left(2 \exp (-r \underline{T})-\exp (-r T)-\frac{P r}{c_{2}}\right)$.

Proof. Note that $\pi_{1}(s)=P\left(1-\frac{c_{1}}{c_{2}}\right)$ for all $s \in[\tilde{s}, T)$ and smaller otherwise and that $\pi_{2}(s)=0$ for all $s \in[\tilde{s}, T]$ and smaller otherwise. Because each starting time contained in the randomization of each player is an optimal strategy against the rival's distribution, the strategy profile is a Nash equilibrium.

Lemma 7. Assume that $\frac{P r}{c_{2}} \leq \exp (-r \underline{T})-\exp (-r T)$. In the unique Nash equilibrium, players randomize their starting times according to the cumulative distribution functions

$$
F_{1}(s)= \begin{cases}0 & \text { for all } 0 \leq s \leq \hat{s} \\ 1-\frac{c_{2}}{P r}(\exp (-r s)-\exp (-r T)) & \text { for all } s \in(\hat{s}, T] \\ 1 & \text { for all } s>T\end{cases}
$$

and

$$
F_{2}(s)= \begin{cases}0 & \text { for all } 0 \leq s \leq \hat{s} \\ \frac{c_{1}}{c_{2}}-\frac{c_{1}}{P r}(\exp (-r s)-\exp (-r T)) & \text { for all } s \in(\hat{s}, T) \\ 1 & \text { for all } s \geq T,\end{cases}
$$

where $\hat{s}=-\frac{1}{r} \log \left(\frac{P r}{c_{2}}+\exp (-r T)\right)$.

Proof. Note that $\pi_{1}(s)=P\left(1-\frac{c_{1}}{c_{2}}\right)$ for all $s \in[\tilde{s}, T)$ and smaller otherwise and that $\pi_{2}(s)=0$ for all $s \in[\tilde{s}, T]$ and smaller otherwise. Because each starting time contained in the randomization of each player is an optimal strategy against the rival's distribution, the strategy profile is a Nash equilibrium.

Step 2 in the proof of Proposition 8: In both Lemma 6 and Lemma 7, the starting distribution of player 2 stochastically dominates the distribution of player 1 . Thus, player 1 exerts a higher expected effort, that is, we can bound the expected effort of player 2 by the expected effort of player 1 . For any duration, player 1 uses the same equilibrium distributions as in the symmetric setting (with $c_{2}=c$ ) and for a short duration, both players use the same equilibrium distributions as in the symmetric setting. Thus, expected effort in the asymmetric setting is the same as in the symmetric setting for a short deadline and lower otherwise. Because a short deadline is optimal in the symmetric setting, it remains optimal in the asymmetric setting. The remainder of the proof for $\underline{T}=0$ and the optimal value of $T$ is identical to the proof in the symmetric setting. 
Proof of Proposition 10. For $\frac{P r}{2 c} \geq 1-\frac{1}{e}$, the optimal solution is still attained for a short deadline and $\underline{T}=0$, because the maximal possible effort is used in the maximization leading to Lemma 4.

For the reverse direction, denote the discounted expected effort for the starting time $\underline{T}=0$ by $\Pi(T)$. Note that for an intermediate duration, we obtain

$$
\begin{aligned}
\Pi(T) & \geq \mathbb{P}\left(\max \left\{\int_{0}^{T} e_{1}^{t} d t, \int_{0}^{T} e_{2}^{t} d t\right\}=T\right) T \exp (-r T) \\
& =\left(2 F(0)-F(0)^{2}\right) T \exp (-r T) \\
& =\frac{4 c}{P r}\left(1-\exp (-r T)-\frac{c}{P r}(1-\exp (-r T))^{2}\right) T \exp (-r T)
\end{aligned}
$$

and that the first inequality holds with equality for $T=-\frac{1}{r} \log \left(1-\frac{P r}{2 c}\right)$, that is, the highest value of $T$ for which both players exert full effort with probability one. Thus,

$$
\begin{aligned}
& \frac{d \Pi}{d T}\left(T=-\frac{1}{r} \log \left(1-\frac{P r}{2 c}\right)\right) \geq \frac{4 c}{P r}((1-r T) \exp (-r T)(1-\exp (-r T) \\
& \left.\left.\left.-\frac{c}{P r}(1-\exp (-r T))^{2}\right)\right)+T \exp (-r T)\left(r \exp (-r T)\left(1-\frac{2 c}{P r}(1-\exp (-r T))\right)\right)\right) .
\end{aligned}
$$

Plugging in $T=-\frac{1}{r} \log \left(1-\frac{P r}{2 c}\right)$, we obtain

$$
\frac{d \Pi}{d T}\left(T=-\frac{1}{r} \log \left(1-\frac{P r}{2 c}\right)\right)=\left(1+\log \left(1-\frac{P r}{2 c}\right)\right)\left(1-\frac{P r}{2 c}\right),
$$

which is positive if $\log \left(1-\frac{P r}{2 c}\right)>0$, that is, if $\frac{P r}{2 c}<1-\frac{1}{e}$. Thus, for $\frac{P r}{2 c}<1-e$, the payoff continues to increase as the parameters switch from a short to an intermediate deadline (and, by continuity, also for some interval of intermediate deadlines). This contradicts optimality of $\underline{T}=0$ and a short deadline. 